\title{
Drivers for the Comorbidity of Type 2 Diabetes Mellitus and Epilepsy: a Scoping Review
}

\author{
Nathan A. Shlobin ${ }^{a}$, Josemir W. Sander ${ }^{b, c}$
}

a Department of Neurological Surgery, Feinberg School of Medicine, Northwestern University, Chicago, IL, USA

${ }^{b}$ NIHR University College London Hospitals Biomedical Research Centre, UCL Queen Square Institute of Neurology, Queen Square, London WC1N 3BG \& Chalfont Centre for Epilepsy, Chalfont St Peter SL9 ORJ, UK

c Stichting Epilepsie Instellingen Nederland (SEIN), Heemstede, The Netherlands

Corresponding author:

Prof Ley Sander, MD PhD FRCP FEAN

Box 29, UCL Queen Square Institute of Neurology

London WC1N 3BG, United Kingdom

E-mail: I.sander@ucl.ac.uk 


\title{
Drivers for the Comorbidity of Type 2 Diabetes Mellitus and Epilepsy: a Scoping Review
}

\begin{abstract}
:
Epilepsy, a common neurologic condition, is associated with a greater prevalence of type 2 diabetes mellitus (T2DM). We examined potential drivers for the comorbidity of epilepsy and T2DM in an attempt to elucidate possible biological mechanisms underlying the development of processes in individuals. We searched PubMed and Medline up to December 2019. Our search yielded 3,361 articles, of which 82 were included in the scoping review. We reviewed articles with a focus on the association of epilepsy and T2DM, drivers, and biological mechanisms. Epilepsy is associated with obesity and obesity is associated with T2DM. Treatment with valproate (either sodium or acid) is associated with weight increase and hyperinsulinemia, while topiramate causes weight loss. People with epilepsy are less likely to exercise which is protective against obesity. Mitochondrial dysfunction and adiponectin deficiency are common to epilepsy and T2DM. One possible mechanism for the comorbidity is mitochondrial dysfunction and adiponectin deficiency which promotes epilepsy, obesity, and T2DM. Another possible mechanism is that people with epilepsy are more likely to be obese due to lack of exercise and the effects of some antiseizure medications, which makes them susceptible to T2DM due to the development of mitochondrial dysfunction and adiponectin deficiency. A third mechanism is that people with epilepsy have greater mitochondrial dysfunction and lower adiponectin levels than people without epilepsy at baseline, which may exacerbate after treatment with antiseizure medications. Future research involving a combined genetic and molecular pathway approach will likely yield valuable insight regarding the comorbidity of epilepsy and T2DM.
\end{abstract}

Keywords: obesity, adiponectin, mitochondrial dysfunction

Declarations of interest: none in relation to this work. This work did not receive any specific grant from funding agencies in the public, commercial, or not-for-profit sectors. 


\section{Introduction}

Epilepsy, a common neurologic symptom-complex contributing to disability and premature mortality, is associated with a greater prevalence of comorbidities [1, 2]. A European cohort study found the cumulative probability of comorbid illness was significantly greater for people with epilepsy than healthy controls and higher seizure frequency associated with a greater number of comorbid conditions [3]. A Norwegian cohort study found that over threequarters of children with epilepsy had one or more comorbid disorders, compared to only about a third of children in the general population [4]. People with drug-refractory epilepsy have higher comorbidity rates than people with controlled epilepsy [5].

A disorder of particular interest is diabetes [6-9]. The connection between epilepsy and type 1 diabetes mellitus (T1DM) has already been highlighted [10-20]. A recent meta-analysis found that people with T1DM have a 2 to 6 fold increased risk of epilepsy than the general population [20]. The onset of T1DM precedes the onset of epilepsy by a mean period of 1.5-2.8 years [20].

Data regarding the comorbid association of epilepsy and type 2 diabetes mellitus (T2DM) are sparser but exist. A cohort study of national health insurance claims in Taiwan found that people with T2DM had a higher incidence of epilepsy than controls independent of severe hypoglycaemia [21]. A Bosnian retrospective study found an association of early and late seizures with T2DM after stroke [22]. Another study also found an increased risk for epilepsy in people with T2DM $[11,23]$. A review confirmed the association between epilepsy and T2DM [24]. Discussions of why this association exist are, however, very incipient. We examine possible drivers for the comorbidity of epilepsy and T2DM in an attempt to elucidate the possible biological mechanisms underlying the development of processes in individuals.

\section{Materials and Methods}

\subsection{Scoping Review}

We carried out a scoping review, used when a narrow review question cannot be determined. Scoping reviews are also useful when studies have employed diverse data collection and analysis methodologies, when no prior synthesis on the topic has been conducted, and the quality of the studies is not assessed [25].

\subsection{Search Strategy}

One of the authors (NS) conducted a search of PubMed and Medline up to December 2019. Search terms were determined after an initial exploration of the literature. These included epilepsy, comorbidity, comorbidities, type 2 diabetes, obesity, and antiepileptic. Boolean operators were also used to combine keywords. There were no language or study design restrictions.

\subsection{Article Screening and Criteria}

Titles and abstract were categorized as relevant or irrelevant. The full text judged as relevant were then evaluated by the reviewer. Articles were included if they were quantitative or qualitative peer-reviewed reports of studies focused on comorbidity of epilepsy with T2DM or obesity, antiseizure medication (ASM) and T2DM or obesity, or the factors associated with 
these conditions; and examined biological mechanisms and/or associations between comorbid conditions.

\subsection{Data Extraction}

Extracted data included the topic, purpose, and type of the study; demographic or other pertinent characteristics; the intervention, if any; the outcomes measured or the processes discussed; and the authors' conclusions.

\subsection{Data Synthesis}

Articles were grouped according to topic. Within each group, numerical data were organized together, and descriptions of biological mechanisms were amalgamated from descriptions in the individual articles.

\section{Results}

Initially, 3,361 articles were identified on PubMed. The keywords were rearranged for greater specificity by including only articles referencing either epilepsy or antiseizure medications along with at least one of T2DM, obesity, and exercise. This still yielded 1,008 articles. An analogous search strategy was employed for Medline, yielding 851 articles (Table 1). Additional searches were made if more information was required on a given topic. Articles were excluded if they did not focus on the comorbidity of epilepsy with T2DM or obesity, factors associated with epilepsy and T2DM or obesity, the effect of antiseizure medication use on factors associated with T2DM or obesity, or cellular or molecular mechanisms associated with either of the three above-mentioned conditions. They were also excluded if the articles were not in peer-reviewed journal, if the abstract or full text could not be sourced, or if they were duplicates. Ultimately, 82 articles were included (Figure 1). These sources included preclinical experiments $(n=7)$, case reports $(n=2)$, correlational studies $(n=13)$, human subjects' experiments $(n=25)$, and reviews $(n=35)$.

\section{Discussion}

\subsection{Epilepsy and Obesity}

An US epidemiological study of a nationally-representative sample of people with epilepsy found adults with any epilepsy (34.1\%) and inactive epilepsy (40.3\%) were more likely to be obese than adults without epilepsy (27.5\%) [7]. This study suggested a possible correlation between epilepsy, and obesity in adults.

A population-based study of children with epilepsy treated at a hospital in Ohio between 2003 and 2006 found children with epilepsy were more likely to be obese than those without [26]. Children with epilepsy who were older, had idiopathic epilepsy, or had no concomitant medication were more likely to be obese than other children with epilepsy [26]. This study suggested a correlation between epilepsy and obesity in children.

A Brazilian cross-sectional study of elderly people whose seizures began over the age of 51 determined that elderly people with new-onset epilepsy had greater waist circumference, indicative of greater levels of abdominal obesity [27]. This suggested a correlation between development of epilepsy and abdominal obesity in elderly people. 
These studies show that epilepsy is frequently comorbid with obesity in children and adults. Possible drivers responsible for the comorbidity of epilepsy and obesity were not discussed in any of the reports [7, 26].

\subsection{Obesity and T2DM}

A review of weight issues in people with epilepsy found that $61 \%$ of the prevalence of T2DM is attributable to obesity and the risk of T2DM doubles with a gain of $5-8 \mathrm{~kg}$ of weight [28]. The likely molecular mechanisms behind T2DM suggest that it is characterized by two main features: insulin resistance and the dysfunction of pancreatic islet $\beta$-cells [29, 30]. Progression from normal glucose tolerance to impaired glucose tolerance is associated with an increase in body weight, decrease in insulin-stimulated glucose disposal, and decrease in the acute insulin secretory response to intravenous glucose. A longitudinal study of Pima Indians demonstrated that the progression from impaired glucose tolerance to diabetes is associated with a further increase in body weight, additional decreases in insulin-stimulated glucose disposal and the acute insulin secretory response [31].

In obese individuals, adipose tissue releases greater amounts of factors such as nonesterified fatty acids, glycerol, hormones, and pro-inflammatory cytokines than in non-obese individuals; triglyceride storage is impaired; and lipolysis is increased [29, 32]. All of these contribute to an increase in circulating fatty acid levels and promote an overload of fatty acids in skeletal muscle and the liver, causing decreased responsiveness of these tissues to insulin in obese individuals [30]. Eventually, this results in insulin resistance [32].

Pancreatic islet $\beta$-cells compensate for insulin resistance by hypersecreting insulin to maintain normoglycemia [33]. T2DM develops in people for which the $\beta$-cell compensatory insulin hypersecretion falters [33]. Insulin levels decrease when fasting glycemia exceeds the upper limit of normal glycemic levels of $5.5 \mathrm{mM}$, indicative of $\beta$-cell failure [34]. $\beta$-cell function is a major determinant of oral glucose tolerance and stimulates the progression from normoglycemia to diabetes [30].

\subsection{ASM Studies in People with Epilepsy}

Studies investigating the physiological effects of antiseizure medication are informative. The enzyme inhibitor valproic acid (VPA) as treatment for epilepsy was associated with weight gain in $50 \%$ of treated women which was associated with hyperinsulinemia [35]. VPA induced a metabolic syndrome consisting of centripetal obesity, hyperinsulinemia, lipid abnormalities, polycystic ovaries, and hyperandrogenism [36]. In a study comparing VPA with the enzyme inducer carbamazepine (CBZ), postprandial insulin and proinsulin levels were significantly higher for people treated with VPA than for people treated with CBZ, while no differences were found in the fasting state [37]. Replacing VPA with lamotrigine (LTG) reduced body mass index (BMI) and fasting serum insulin and testosterone concentrations and increased high density lipoprotein cholesterol to total cholesterol ratios [36]. Levels of these compounds returned to normal within two months of starting LTG [36].

A study of people with epilepsy treated with topiramate for one year found that up to $86 \%$ had reductions in baseline weight at 1 year [38]. Obese people had higher reductions in baseline weight than those non-obese [38]. Apart from its original antiseizure properties, topiramate has been used as a weight loss drug. It was found to decrease blood glucose levels 
by $16 \%$ and insulin levels by $24 \%$, in obese individuals but had no effect on serum glucose, insulin or triglyceride levels in non-obese people [39]. Overall, topiramate led to the largest weight loss in people with a high BMI [40]. In people with T2DM, weight loss and BMI reduction were higher in the topiramate treatment group than in controls [41]. These studies suggest baseline BMI predicts weight loss after topiramate treatment, particularly in people with epilepsy.

\subsection{Role of Exercise}

People with epilepsy are less likely to engage or even intend to engage in physical activities [42-44]. In a Norwegian sample, the proportion of people never exercising was significantly greater for an epilepsy cohort than the average population [45]. The physiological parameter of interest is the modifiable risk factor cardiorespiratory fitness, which is lower in people with epilepsy than age and gender-matched controls [46]. People with epilepsy have often been discouraged from partaking in physical activity due to concerns about inducing or increasing the frequency of seizures [43]. Similarly, interventions designed to increase exercise in people with epilepsy often fail to initially increase exercise or sustain increased exercise [47]. Reduced levels of exercise in people with epilepsy may result from depression, anxiety, perceived stigma, or the side effects of epileptic drugs [48].

Preclinical and human studies have shown that exercise is beneficial for people with epilepsy. In rat models of temporal lobe epilepsy and absence epilepsy, the exercise group experienced a significant reduction in seizure frequency compared to controls and sham groups $[49,50]$. Studies conducted using a rat pentylenetetrazole model determined that exercise decreased seizure susceptibility, frequency, and latency [51, 52].

In a human trial, fifteen women with drug-resistant epilepsy engaged in aerobic dancing with strength training and stretching for one hour twice weekly for 15 weeks [53]. The frequency of self-reported seizures significantly decreased during the exercise intervention period [53]. A Chinese study using an intervention consisting of a low glycaemic diet and structured exercise decreased seizure frequency in children with refractory epilepsy [54]. A randomized control trial found physical activity decreased seizure frequency in children with epilepsy aged 8-14 [55]. This may result physiologically from reduced epileptic discharges during exercise [56], providing neuroprotective effects such as improved seizure control and improved cognitive function [5761].

Exercise also affects the onset of epilepsy. A Swedish cohort study examining individuals initially free of epilepsy who participated in the cross-country Vasaloppet ski race compared to non-participating matched controls found that exercise decreased the incidence of epilepsy, indicating physical activity may delay or prevent epilepsy [62]. The intensity of exercise inversely correlated with incidence of epilepsy [62]. Lack of exercise may initially predispose people to epilepsy, and continued lack of exercise may allow for continued occurrence of seizures.

\subsection{Mitochondrial Dysfunction}

Mitochondrial dysfunction is common to epilepsy, obesity, and diabetes. Epilepsy is a neurological and systemic disorder resulting from fundamental pathophysiological processes [63]. Mitochondrial dysfunction is one of these processes and is linked to epilepsy through 
changes in ATP production, calcium homeostasis, and oxidative stress by reactive oxygen species (ROS) [64]. Mitochondrial oxidative phosphorylation is the primary source of ATP production in neurons [65]. Mutations in mitochondrial DNA or nuclear genes resulting in impairment of the respiratory chain or mitochondrial ATP synthesis, are associated with epilepsy [66]. People with respiratory chain dysfunction may have epilepsy associated with an encephalopathy. As an example of a mitochondrial encephalopathy, deficiency of monosialodihexosylganglioside (GM3) synthase, an enzyme involved in the synthesis of complex gangliosides, results in secondary respiratory chain dysfunction and early-onset epilepsy [67]. Mitochondria are also involved in cellular calcium homeostasis [65]. Deficiencies in proper calcium regulation are associated with epilepsy [64]. Mitochondria are also the main site of ROS production, making them vulnerable to oxidative stress [68, 69]. Dysfunction resulting from oxidative stress promotes neuronal hyperexcitability by decreasing membrane potential and reducing network inhibition and alters synaptic transmission by damaging ion channels and neurotransmitter transports, all of which increase seizure susceptibility $[64,65$, 68-70]. High oxidative status is associated with severity and recurrence of epileptic seizures [71]. Mitochondrial dysfunction also triggers neuronal cell death, a hallmark of drug-refractory epilepsy [65]. In mesial temporal lobe epilepsy with hippocampal sclerosis, progressive loss of pyramidal cells in the CA1, CA3, and CA4 layers occurs, correlated with impairment of respiratory chain complex I activity [72].

Seizures acutely necessitate excessive energy from the brain and are a manifestation of impaired mitochondrial energy production [64, 72]. Molecularly, this is associated with a decreased phosphocreatine/adenosine triphosphate (PCr/ATP) ratio [72]. The ketogenic diet has been associated with improved mitochondrial energy production via upregulation of mitochondrial genes and increased mitochondrial biogenesis in animal models [72]. In a MRspectroscopy study of people with intractable epilepsy, the ratio of PCr/ATP and PCr/Pi significantly increased after a ketogenic diet compared to baseline, indicating an improvement in cerebral energy metabolism [72]. A calorie-restricted diet is less restrictive than a ketogenic diet but may reduce seizure frequency by improving mitochondrial energy production in the same way as a ketogenic diet [73]. It has also been hypothesized that pharmacoresistance and the subsequent likelihood of seizure recurrence results from the interplay of the seizuregenic potential of the epileptic focus, efficacy of ASMs, and mitochondrial dysfunction [72]. Preclinical and clinical studies are necessary to validate this hypothesis.

Mitochondrial dysfunction is linked to obesity and T2DM through its effects on ATP production, calcium homeostasis, and ROS generation [74-78]. Rates of mitochondrial oxidative phosphorylation in skeletal muscle were $30 \%$ less in insulin-resistant individuals than in controls [72]. suggesting deficiencies in mitochondrial ATP production [75]. In obese human and animal models, deficiencies in calcium homeostasis in the liver, adipocytes, and macrophages occur due to increased physical interaction and functional coupling between the endoplasmic reticulum and mitochondria and calcium transport [76]. Excessive caloric intake increases ROS production [74]. These factors allow for the development of obesity and T2DM. Mitochondrial dysfunction in T2DM leads to insulin resistance through deficiencies in insulin responsiveness of skeletal muscle and liver, mainly due to lower mitochondrial biogenesis and possibly reduced functional capacity per mitochondrion $[75,77,79]$. In an obese rat model, hepatic mitochondrial dysfunction preceded insulin resistance [73]. Insulin resistance results from dysregulation of 
fatty acid metabolism in muscle cells through lack of ATP production and dysregulation of calcium homeostasis [75, 76]. Mitochondrial dysfunction also leads to dysfunction of pancreatic islet $\beta$-cells through increased lipid levels, hyperglycemia, and obesity [77]. ROS accumulation blocks fatty acid oxidation, resulting in lipid accumulation [74]. ROS accumulation also leads to deficiencies in glucose metabolism, namely insulin resistance in 3T3L1 pre-adipocytes [74]. Mitochondrial dysfunction leads to epilepsy, obesity, and T2DM through the same general mechanisms. Exercise reduces mitochondrial dysfunction [80-82], and may be protective against epilepsy, obesity, and T2DM.

\subsection{Adiponectin Deficiency}

Adiponectin stimulates fatty acid oxidation and glucose uptake in skeletal muscle [83], Epilepsy is associated with adiponectin deficiency through the anti-inflammatory effects and wide-ranging CNS effects of adiponectin [84]. People with temporal lobe epilepsy may have lower circulating adiponectin levels than healthy people [84]. Epilepsy duration in temporal lobe epilepsy seems inversely associated with adiponectin plasma levels [84]. Adiponectin increases within 24 hours of tonic-clonic seizures but decreases in the interictal period, indicating the presence of a brief anti-inflammatory state within the setting of a persistent pro-inflammatory condition $[84,85]$. The immediate rise in adiponectin levels following convulsions presumably occurs to prevent deleterious CNS effects [85]. Continuous activation of the CNS, particularly astrocytes and microglia, secondary to long-term adiponectin deficiency may enhance local and systemic inflammatory mechanisms [84].

Adiponectin deficiency also often occurs after ASM treatment. In a study of people treated with VPA, about a third developed obesity, exhibiting circulating insulin levels significantly higher and adiponectin levels significantly lower than those who did not gain weight [86]. Overweightness associated with VPA is accompanied by lower adiponectin levels [87]. Similarly, treatment with VPA predisposed obese children with idiopathic epilepsy to insulin resistance and lower adiponectin levels compared to age and gender matched obese controls [88]. VPA also induced hypoadiponectinemia and insulin resistance in previously untreated people with epilepsy $[89,90]$. Other studies, however, have found non-significant increases in adiponectin levels after VPA treatment $[91,92]$. Children with epilepsy treated with topiramate showed increased serum levels of adiponectin and weight loss, suggesting the association between levels of adiponectin and weight loss mediated by topiramate [93].

Low plasma adiponectin levels are associated with obesity and T2DM [83, 94]. The degree of hypoadiponectinemia is most closely associated with the degree of insulin resistance and hyperinsulinemia $[89,93]$. The presence of insulin resistant previously untreated females with epilepsy indicates that weight gain itself may not cause insulin resistance [89], but rather that adiponectin deficiency may promote insulin resistance and weight gain. While R112C, 1164T, R221S, and H241P are missense mutations in adiponectin, the frequency of the 1164T mutation is significantly higher in people with T2DM than controls, and all subjects with the 1164T mutation had T2DM [95].

A preclinical study has suggested a possible connection between epilepsy, obesity, and T2DM involving adiponectin deficiency. Mice with adiponectin deficiency fed a high-fat diet had greater fat accumulation, hyperlipidemia, impaired glucose tolerance, and increased kainic acidinduced seizure severity [96]. A lack of adiponectin has systemic effects, precipitating all three 
conditions. Exercise increases adiponectin levels in preclinical and human studies [97-102], and may be protective against epilepsy, obesity, and T2DM.

\subsection{Proposed Mechanisms for Development of T2DM in People with Epilepsy}

One possible mechanism that we propose for the development of T2DM in people with epilepsy is that mitochondrial dysfunction [63-79] and adiponectin deficiency [83-102] predispose people to epilepsy, obesity, and diabetes. The same biological drivers underlie each of these three conditions due to the systemic nature of both these drivers and the conditions. Inadequate exercise can contribute to these biological drivers [80-82, 97-102]. Temporally, the precise sequence of comorbidity development may be highly individualized: some people may develop epilepsy and then T2DM or vice versa. The degree of mitochondrial dysfunction and adiponectin deficiency and local and systemic contributions of these drivers relative to each other may underlie which condition develops first.

Another possible mechanism is that people with epilepsy are more likely to be obese as they exercise less $[43,45,49,53]$. They are also more likely to be obese if treated with VPA [35-37], contributing to mitochondrial dysfunction and adiponectin deficiency. Obesity, in turn, predisposes them to T2DM [28]. Mitochondrial dysfunction and adiponectin deficiency develop in people with obesity as they continue living with the condition. These pathophysiological processes may become apparent prior to development of T2DM in people with epilepsy, and their further progression may precipitate T2DM.

A third possible mechanism is that, at baseline, people with epilepsy have greater mitochondrial dysfunction and lower adiponectin levels than people without epilepsy, and treatment with ASMs lowers adiponectin levels and increases mitochondrial dysfunction further compared to people without epilepsy [64, 72, 84, 87-93]. Mitochondrial dysfunction and adiponectin deficiency in people with epilepsy precipitate the development of obesity, T2DM, or both conditions. Whether people develop obesity, T2DM, or both and the time elapsed between epilepsy onset and development of these conditions may depend on the absolute or relative degrees of mitochondrial dysfunction and adiponectin deficiency.

All three mechanisms are likely involved in the comorbidity of epilepsy and T2DM. The shared biological drivers of epilepsy and T2DM may occur either sequentially or concurrently. More research is warranted to determine factors associated with each of these mechanisms.

\section{Conclusions}

The association between epilepsy and T2DM is not yet clear and suffers from a lack of epidemiological evidence and sustained biomedical research efforts. We propose three possible mechanisms for the comorbidity of epilepsy and T2DM. One mechanism is that mitochondrial dysfunction and adiponectin deficiency promote epilepsy, obesity, and T2DM. The degree of each of these pathogenic changes determines which condition develops first. Another mechanism is that people with epilepsy are more likely to be obese due to lack of exercise and the effects of some ASMs, which makes them susceptible to T2DM due to the development of mitochondrial dysfunction and adiponectin deficiency. A third mechanism is that people with epilepsy have greater mitochondrial dysfunction and lower adiponectin levels than people without epilepsy at baseline, which is exacerbated after treatment with ASMs. The absolute or 
relative degrees of mitochondrial dysfunction and adiponectin deficiency may affect whether and when people develop obesity, T2DM, or both conditions.

More work is required to determine the precise association between epilepsy and T2DM and whether these proposed mechanisms are valid. One technique that can be used to accomplish this is genome-wide association studies. Genome-wide association studies have characterized genes involved in the development of T2DM [103-107]. Screening for epilepsy and T2DM will be useful in delineating genes implicated in both conditions. Genome-wide association studies likely to be complicated by the interactions of different combinations of loci in epilepsy, which produce pathogenic and clinical heterogeneity [108]. Another technique is exhaustively characterizing the molecular pathways resulting in each condition [109]. This technique, however, provides only indirect evidence for comorbidities as it relies on molecular interaction networks [109], which are often difficult to elucidate. A novel approach integrating genetic information and high-level molecular changes has demonstrated strong consistency with known comorbid disease [109]. Using a combined genetic and molecular pathway approach for epilepsy and T2DM will likely yield valuable insight regarding the comorbidity of these conditions.

\section{Acknowledgements}

JWS is based at UCLH/UCL Comprehensive Biomedical Research Centre, which receives a proportion of funding from the UK Department of Health's NIHR Biomedical Research Centres funding scheme. He receives support from the Dr. Marvin Weil Epilepsy Research Fund, the Christelijke Verenigingvoor de Verpleging van Lijdersaan Epilepsie, Netherlands, and UK Epilepsy Society.

\section{Disclosures}

NAS reports no disclosures in relation to the manuscript. JWS reports personal fees from Eisai, UCB and Zogenix, grants from Eisai, UCB and GW Phama, outside the submitted work. 


\section{References}

[1] Organization WH. Epilepsy in the WHO Africa Region: Bridging the Gap: The Global Campaign Against Epilepsy: "Out of the Shadows." World Health Organization Regional Office for Africa 2004.

[2] Ngugi AK, Bottomley C, Kleinschmidt I, Sander JW, Newton CR. Estimation of the burden of active and life-time epilepsy: a meta-analytic approach. Epilepsia 2010;51: 883-890.

[3] Van Den Broek M, Group* EBftR. Morbidity in patients with epilepsy: type and complications: a European cohort study. Epilepsia 2004;45: 71-76.

[4] Aaberg KM, Bakken IJ, Lossius MI, Søraas CL, Håberg SE, Stoltenberg C, Surén P, Chin R. Comorbidity and childhood epilepsy: a nationwide registry study. Pediatrics 2016;138: e20160921.

[5] Cramer JA, Wang ZJ, Chang E, Powers A, Copher R, Cherepanov D, Broder MS. Healthcare utilization and costs in adults with stable and uncontrolled epilepsy. Epilepsy \& behavior 2014;31: 356-362.

[6] Ottman R, Lipton RB, Ettinger AB, Cramer JA, Reed ML, Morrison A, Wan GJ. Comorbidities of epilepsy: results from the Epilepsy Comorbidities and Health (EPIC) survey. Epilepsia 2011;52: 308-315.

[7] Kadima NT, Kobau R, Zack MM, Helmers S. Comorbidity in adults with epilepsy-United States, 2010. MMWR. Morbidity and mortality weekly report 2013;62: 849.

[8] Gaitatzis A, Carroll K, Majeed A, Sander JW. The epidemiology of the comorbidity of epilepsy in the general population. Epilepsia 2004;45: 1613-1622.

[9] Rai D, Kerr MP, McManus S, Jordanova V, Lewis G, Brugha TS. Epilepsy and psychiatric comorbidity: a nationally representative population-based study. Epilepsia 2012;53: 1095-1103.

[10] McCorry D, Nicolson A, Smith D, Marson A, Feltbower RG, Chadwick D. An association between type 1 diabetes and idiopathic generalized epilepsy. Annals of neurology 2006;59: 204206.

[11] O'Connell MA, Harvey AS, Mackay MT, Cameron FJ. Does epilepsy occur more frequently in children with Type 1 diabetes? Journal of paediatrics and child health 2008;44: 586-589.

[12] Mancardi MM, Striano P, Giannattasio A, Baglietto MG, Errichiello L, Zara F, Prato G, Minuto N, Veneselli E, Lorini R. Type 1 diabetes and epilepsy: more than a casual association? Epilepsia 2010;51: 320-321.

[13] Ramakrishnan R, Appleton R. Study of prevalence of epilepsy in children with type 1 diabetes mellitus. Seizure 2012;21: 292-294.

[14] Schober E, Otto KP, Dost A, Jorch N, Holl R. Association of epilepsy and type 1 diabetes mellitus in children and adolescents: is there an increased risk for diabetic ketoacidosis? The Journal of pediatrics 2012;160: 662-666. e1.

[15] Keezer MR, Novy J, Sander JW. Type 1 diabetes mellitus in people with pharmacoresistant epilepsy: prevalence and clinical characteristics. Epilepsy research 2015;115: 55-57.

[16] Chou I-C, Wang C-H, Lin W-D, Tsai F-J, Lin C-C, Kao C-H. Risk of epilepsy in type 1 diabetes mellitus: a population-based cohort study. Diabetologia 2016;59: 1196-1203.

[17] Dafoulas GE, Toulis KA, Mccorry D, Kumarendran B, Thomas GN, Willis BH, Gokhale K, Gkoutos G, Narendran P, Nirantharakumar K. Type 1 diabetes mellitus and risk of incident epilepsy: a population-based, open-cohort study. Diabetologia 2017;60: 258-261.

[18] Ong M-S, Kohane IS, Cai T, Gorman MP, Mandl KD. Population-level evidence for an autoimmune etiology of epilepsy. JAMA neurology 2014;71: 569-574.

[19] Yan D, Zhao E, Zhang H, Luo X, Du Y. Association between type 1 diabetes mellitus and risk of epilepsy: a meta-analysis of observational studies. Drug discoveries \& therapeutics 2017;11: 146-151. 
[20] Mastrangelo M, Tromba V, Silvestri F, Costantino F. Epilepsy in children with type 1 diabetes mellitus: Pathophysiological basis and clinical hallmarks. European Journal of Paediatric Neurology 2018.

[21] Lu C-L, Chang Y-H, Sun Y, Li C-Y. A population-based study of epilepsy incidence in association with type 2 diabetes and severe hypoglycaemia. Diabetes research and clinical practice 2018;140: 97-106.

[22] Bratiæ M, Suljiæ E, Hrnjica M, Resiæ H. Correlation between early and late epileptic seizures and diabetes mellitus during and after stroke. Diabetologia Croatica 2002;31: 3 .

[23] Klimek P, Kautzky-Willer A, Chmiel A, Schiller-Frühwirth I, Thurner S. Quantification of diabetes comorbidity risks across life using nation-wide big claims data. PLoS computational biology 2015;11: e1004125.

[24] Yun C, Xuefeng W. Association between seizures and diabetes mellitus: a comprehensive review of literature. Current diabetes reviews 2013;9: 350-354.

[25] Arksey H, O'Malley L. Scoping studies: towards a methodological framework. International journal of social research methodology 2005;8: 19-32.

[26] Daniels Z, Nick T, Liu C, Cassedy A, Glauser T. Obesity is a common comorbidity for pediatric patients with untreated, newly diagnosed epilepsy. Neurology 2009;73: 658-664.

[27] Tedrus GMA, Srebernich SM, Santos TBN. Correlation between clinical and cognitive aspects and nutritional indicators of elderly patients with new-onset epilepsy. Epilepsy \& Behavior 2018;85: 105-109.

[28] Ben-Menachem E. Weight issues for people with epilepsy-a review. Epilepsia 2007;48: 42-45.

[29] Kahn SE, Hull RL, Utzschneider KM. Mechanisms linking obesity to insulin resistance and type 2 diabetes. Nature 2006;444: 840.

[30] Kahn S. The relative contributions of insulin resistance and beta-cell dysfunction to the pathophysiology of type 2 diabetes. Diabetologia 2003;46: 3-19.

[31] Weyer C, Bogardus C, Mott DM, Pratley RE. The natural history of insulin secretory dysfunction and insulin resistance in the pathogenesis of type 2 diabetes mellitus. The Journal of clinical investigation 1999;104: 787-794.

[32] Guilherme A, Virbasius JV, Puri V, Czech MP. Adipocyte dysfunctions linking obesity to insulin resistance and type 2 diabetes. Nature reviews Molecular cell biology 2008;9: 367.

[33] Prentki M, Nolan CJ. Islet $\beta$ cell failure in type 2 diabetes. The Journal of clinical investigation 2006;116: 1802-1812.

[34] Leahy JL. Pathogenesis of type 2 diabetes mellitus. Archives of medical research 2005;36: 197-209.

[35] Isojärvi JI, Laatikainen TJ, Knip M, Pakarinen AJ, Juntunen KT, Myllyla VV. Obesity and endocrine disorders in women taking valproate for epilepsy. Annals of Neurology: Official Journal of the American Neurological Association and the Child Neurology Society 1996;39: 579-584.

[36] Isojärvi JI, Rättyä J, Myllylä VV, Knip M, Koivunen R, Pakarinen AJ, Tekay A, Tapanainen JS. Valproate, lamotrigine, and insulin-mediated risks in women with epilepsy. Annals of neurology 1998;43: 446-451.

[37] Luef G, Abraham I, Haslinger M, Trinka E, Seppi K, Unterberger I, Alge A, Windisch J, Lechleitner M, Bauer G. Polycystic ovaries, obesity and insulin resistance in women with epilepsy. Journal of neurology 2002;249: 835-841.

[38] Ben-Menachem E, Axelsen M, Johanson EH, Stagge A, Smith U. Predictors of weight loss in adults with topiramate-treated epilepsy. Obesity research 2003;11: 556-562.

[39] Smith U, Axelsen M, Hellebo-Johanson E, Lundgren B, Ben-Menachem E. Topiramate, a novel antiepileptic drug, reduces body weight and food intake in obesity. In: Obesity Research: NORTH AMER ASSOC STUDY OBESITY C/O DR MICHAEL JENSEN, MAYO MEDICAL CENTER ...; 2000. p. 10S-10S. 
[40] Verrotti A, Scaparrotta A, Agostinelli S, Di Pillo S, Chiarelli F, Grosso S. Topiramateinduced weight loss: a review. Epilepsy research 2011;95: 189-199.

[41] Moradi S, Kerman SRJ, Mollabashi M. The effect of topiramate on weight loss in patients with type 2 diabetes. Journal of research in medical sciences: the official journal of Isfahan University of Medical Sciences 2013;18: 297.

[42] Fernandez H, Cooke M, Patel T. Epilepsy and lifestyle behaviors related to bone health. Epilepsia 2019.

[43] Arida RM, Cavalheiro EA, da Silva AC, Scorza FA. Physical activity and epilepsy. Sports Medicine 2008;38: 607-615.

[44] Vancampfort D, Ward PB, Stubbs B. Physical activity and sedentary levels among people living with epilepsy: A systematic review and meta-analysis. Epilepsy Behav 2019;99: 106390.

[45] Nakken KO. Clinical research physical exercise in outpatients with epilepsy. Epilepsia 1999;40: 643-651.

[46] Vancampfort D, Ward PB, Stubbs B. Physical fitness levels and moderators in people with epilepsy: A systematic review and meta-analysis. Epilepsy Behav 2019;99: 106448.

[47] Brown DM, Mahlberg N, Pohl D, Timmons BW, Bray SR, Streiner DL, Ferro MA, Hamer $\mathrm{S}$, Rosenbaum PL, Ronen GM. Can behavioral strategies increase physical activity and influence depressive symptoms and quality of life among children with epilepsy? Results of a randomized controlled trial. Epilepsy \& Behavior 2019;94: 158-166.

[48] Vancampfort D, Ward PB. Physical activity correlates across the lifespan in people with epilepsy: a systematic review. Disability and Rehabilitation 2019: 1-8.

[49] Arida RM, Scorza FA, dos Santos NF, Peres CA, Cavalheiro EA. Effect of physical exercise on seizure occurrence in a model of temporal lobe epilepsy in rats. Epilepsy research 1999;37: 45-52.

[50] Aygun H, Ayyildiz M, Agar E. Swimming exercise decreases the absence-like epileptic activity in WAG/Rij rats. Behavioural brain research 2019;363: 145-148.

[51] Barzroodi Pour M, Bayat M, Golab F, Eftekharzadeh M, Katebi M, Soleimani M, Karimzadeh $F$. The effect of exercise on GABA signaling pathway in the model of chemically induced seizures. Life Sci 2019;232: 116667.

[52] Lin X-Y, Cui Y, Wang L, Chen W. Chronic exercise buffers the cognitive dysfunction and decreases the susceptibility to seizures in PTZ-treated rats. Epilepsy \& Behavior 2019;98: 173187.

[53] Eriksen HR, Ellertsen B, Grønningsæter H, Nakken KO, Løyning Y, Ursin H. Physical exercise in women with intractable epilepsy. Epilepsia 1994;35: 1256-1264.

[54] Zhang H, Yu L, Li H, Liu Y. Effect of low glycaemic diet and structured exercise on quality of life and psychosocial functions in children with epilepsy. J Int Med Res 2019: 300060519893855.

[55] Willis J, Hophing L, Mahlberg N, Ronen GM. Youth with epilepsy: Their insight into participating in enhanced physical activity study. Epilepsy \& Behavior 2018;89: 63-69.

[56] Carrizosa-Moog J, Ladino LD, Benjumea-Cuartas V, Orozco-Hernández JP, CastrillónVelilla DM, Rizvi S, Téllez-Zenteno JF. Epilepsy, Physical Activity and Sports: A Narrative Review. Canadian Journal of Neurological Sciences 2018;45: 624-632.

[57] Pimentel J, Tojal R, Morgado J. Epilepsy and physical exercise. Seizure 2015;25: 87-94.

[58] Arida RM, de Almeida A-CG, Cavalheiro EA, Scorza FA. Experimental and clinical findings from physical exercise as complementary therapy for epilepsy. Epilepsy \& behavior 2013;26: 273-278.

[59] Arida RM, Scorza FA, Cavalheiro EA. Favorable effects of physical activity for recovery in temporal lobe epilepsy. Epilepsia 2010;51: 76-79. 
[60] Mario Arida R, Alexandre Scorza F. Role of physical exercise as complementary treatment for epilepsy and other brain disorders. Current pharmaceutical design 2013;19: 67206725.

[61] Allendorfer JB, Arida RM. Role of physical activity and exercise in alleviating cognitive impairment in people with epilepsy. Clinical therapeutics 2018;40: 26-34.

[62] Ahl M, Avdic U, Strandberg MC, Chugh D, Andersson E, Hållmarker U, James S, Deierborg T, Ekdahl CT. Physical Activity Reduces Epilepsy Incidence: a Retrospective Cohort Study in Swedish Cross-Country Skiers and an Experimental Study in Seizure-Prone Synapsin II Knockout Mice. Sports Medicine-Open 2019;5: 52.

[63] Yuen AW, Keezer MR, Sander JW. Epilepsy is a neurological and a systemic disorder. Epilepsy \& Behavior 2018;78: 57-61.

[64] Zsurka G, Kunz WS. Mitochondrial dysfunction and seizures: the neuronal energy crisis. The Lancet Neurology 2015;14: 956-966.

[65] Folbergrová J, Kunz WS. Mitochondrial dysfunction in epilepsy. Mitochondrion 2012;12: 35-40.

[66] Kudin AP, Zsurka G, Elger CE, Kunz WS. Mitochondrial involvement in temporal lobe epilepsy. Experimental neurology 2009;218: 326-332.

[67] Fragaki K, Ait-El-Mkadem S, Chaussenot A, Gire C, Mengual R, Bonesso L, Bénéteau M, Ricci J-E, Desquiret-Dumas V, Procaccio V. Refractory epilepsy and mitochondrial dysfunction due to GM3 synthase deficiency. European Journal of Human Genetics 2013;21: 528.

[68] Waldbaum S, Patel M. Mitochondria, oxidative stress, and temporal lobe epilepsy. Epilepsy research 2010;88: 23-45.

[69] Patel M. Mitochondrial dysfunction and oxidative stress: cause and consequence of epileptic seizures. Free Radical Biology and Medicine 2004;37: 1951-1962.

[70] Waldbaum S, Patel M. Mitochondrial dysfunction and oxidative stress: a contributing link to acquired epilepsy? Journal of bioenergetics and biomembranes 2010;42: 449-455.

[71] Chang S-J, Yu B-C. Mitochondrial matters of the brain: mitochondrial dysfunction and oxidative status in epilepsy. Journal of bioenergetics and biomembranes 2010;42: 457-459.

[72] Yuen AW, Sander JW. Impaired mitochondrial energy production: the basis of pharmacoresistance in epilepsy. Medical hypotheses 2011;77: 536-540.

[73] Yuen AW, Sander JW. Rationale for using intermittent calorie restriction as a dietary treatment for drug resistant epilepsy. Epilepsy \& Behavior 2014;33: 110-114.

[74] Bournat JC, Brown CW. Mitochondrial dysfunction in obesity. Current opinion in endocrinology, diabetes, and obesity 2010;17: 446.

[75] Petersen KF, Dufour S, Befroy D, Garcia R, Shulman GI. Impaired mitochondrial activity in the insulin-resistant offspring of patients with type 2 diabetes. New England Journal of Medicine 2004;350: 664-671.

[76] Arruda AP, Hotamisligil GS. Calcium homeostasis and organelle function in the pathogenesis of obesity and diabetes. Cell metabolism 2015;22: 381-397.

[77] Lowell BB, Shulman GI. Mitochondrial dysfunction and type 2 diabetes. Science 2005;307: 384-387.

[78] Rector RS, Thyfault JP, Uptergrove GM, Morris EM, Naples SP, Borengasser SJ, Mikus $\mathrm{CR}$, Laye MJ, Laughlin MH, Booth FW. Mitochondrial dysfunction precedes insulin resistance and hepatic steatosis and contributes to the natural history of non-alcoholic fatty liver disease in an obese rodent model. Journal of hepatology 2010;52: 727-736.

[79] Højlund K, Mogensen M, Sahlin K, Beck-Nielsen H. Mitochondrial dysfunction in type 2 diabetes and obesity. Endocrinology and metabolism clinics of North America 2008;37: 713731. 
[80] Tsai H-H, Chang S-C, Chou C-H, Weng T-P, Hsu C-C, Wang J-S. Exercise training alleviates hypoxia-induced mitochondrial dysfunction in the lymphocytes of sedentary males. Scientific reports $2016 ; 6: 35170$.

[81] Joseph AM, Adhihetty PJ, Leeuwenburgh C. Beneficial effects of exercise on agerelated mitochondrial dysfunction and oxidative stress in skeletal muscle. The Journal of physiology 2016;594: 5105-5123.

[82] Heo J-W, No M-H, Park D-H, Kang J-H, Seo DY, Han J, Neufer PD, Kwak H-B. Effects of exercise on obesity-induced mitochondrial dysfunction in skeletal muscle. The Korean Journal of Physiology \& Pharmacology 2017;21: 567-577.

[83] Yamauchi T, Kamon J, Ito Y, Tsuchida A, Yokomizo T, Kita S, Sugiyama T, Miyagishi M, Hara K, Tsunoda M. Cloning of adiponectin receptors that mediate antidiabetic metabolic effects. Nature 2003;423: 762.

[84] de Brito Toscano EC, Lessa JMK, Gonçalves AP, Rocha NP, Giannetti AV, de Oliveira GN, Rachid MA, Vieira ÉLM, Teixeira AL. Circulating levels of adipokines are altered in patients with temporal lobe epilepsy. Epilepsy \& Behavior 2019;90: 137-141.

[85] Palmio J, Vuolteenaho K, Lehtimäki K, Nieminen R, Peltola J, Moilanen E. CSF and plasma adipokines after tonic-clonic seizures. Seizure 2016;39: 10-12.

[86] Greco R, Latini G, Chiarelli F, lannetti P, Verrotti A. Leptin, ghrelin, and adiponectin in epileptic patients treated with valproic acid. Neurology 2005;65: 1808-1809.

[87] Rauchenzauner M, Haberlandt E, Scholl-Bürgi S, Ernst B, Hoppichler F, Karall D, Ebenbichler CF, Rostasy K, Luef G. Adiponectin and visfatin concentrations in children treated with valproic acid. Epilepsia 2008;49: 353-357.

[88] Aly R, Amr N, Saad W, Megahed A. Insulin resistance in patients on valproic acid: relation to adiponectin. Acta Neurologica Scandinavica 2015;131: 169-175.

[89] Sidhu HS, Srinivas R, Sadhotra A. Evaluate the effects of long-term valproic acid treatment on metabolic profiles in newly diagnosed or untreated female epileptic patients: a prospective study. Seizure 2017;48: 15-21.

[90] Nisha Y, Bobby Z, Wadwekar V. Biochemical derangements related to metabolic syndrome in epileptic patients on treatment with valproic acid. Seizure 2018;60: 57-60.

[91] Tokgoz H, Aydin K, Oran B, Kiyici A. Plasma leptin, neuropeptide Y, ghrelin, and adiponectin levels and carotid artery intima media thickness in epileptic children treated with valproate. Child's Nervous System 2012;28: 1049-1053.

[92] Sonmez FM, Zaman D, Aksoy A, Deger O, Aliyazicioglu R, Karaguzel G, Fazlioglu K. The effects of topiramate and valproate therapy on insulin, c-peptide, leptin, neuropeptide $Y$, adiponectin, visfatin, and resistin levels in children with epilepsy. Seizure 2013;22: 856-861. [93] Li Hf, Zou Y, Xia Zz, Gao F, Feng Jh, Yang Cw. Effects of topiramate on weight and metabolism in children with epilepsy. Acta Paediatrica 2009;98: 1521-1525.

[94] Weyer C, Funahashi T, Tanaka S, Hotta K, Matsuzawa Y, Pratley RE, Tataranni PA. Hypoadiponectinemia in obesity and type 2 diabetes: close association with insulin resistance and hyperinsulinemia. The Journal of Clinical Endocrinology \& Metabolism 2001;86: 1930-1935. [95] Kondo H, Shimomura I, Matsukawa Y, Kumada M, Takahashi M, Matsuda M, Ouchi N, Kihara S, Kawamoto T, Sumitsuji S. Association of adiponectin mutation with type 2 diabetes: a candidate gene for the insulin resistance syndrome. Diabetes 2002;51: 2325-2328.

[96] Lee EB, Warmann G, Dhir R, Ahima RS. Metabolic dysfunction associated with adiponectin deficiency enhances kainic acid-induced seizure severity. Journal of Neuroscience 2011;31: 14361-14366.

[97] Su M, Bai Y, Song W, Wang M, Shen R, Du K, Xia X, Nie L. Effect of exercise on adiponectin in aged obese rats. Zhongguo ying yong sheng li xue za zhi=Zhongguo yingyong shenglixue zazhi= Chinese journal of applied physiology 2018;34: 345-349. 
[98] Jamurtas AZ, Stavropoulos-Kalinoglou A, Koutsias S, Koutedakis Y, Fatouros I. Adiponectin, resistin, and visfatin in childhood obesity and exercise. Pediatric exercise science 2015;27: 454-462.

[99] Wang X, You T, Murphy K, Lyles MF, Nicklas BJ. Addition of exercise increases plasma adiponectin and release from adipose tissue. Medicine and science in sports and exercise 2015;47: 2450 .

[100] Jürimäe J, Hofmann P, Jürimäe T, Mäestu J, Purge P, Wonisch M, Pokan R, Von Duvillard S. Plasma adiponectin response to sculling exercise at individual anaerobic threshold in college level male rowers. International journal of sports medicine 2006;27: 272-277. [101] Jürimäe J, Purge $P$, Jürimäe $T$. Adiponectin is altered after maximal exercise in highly trained male rowers. European journal of applied physiology 2005;93: 502-505.

[102] Numao S, Katayama Y, Hayashi Y, Matsuo T, Tanaka K. Influence of acute aerobic exercise on adiponectin oligomer concentrations in middle-aged abdominally obese men. Metabolism 2011;60: 186-194.

[103] Saxena R, Voight BF, Lyssenko V, Burtt NP, de Bakker PI, Chen H, Roix JJ, Kathiresan S, Hirschhorn JN, Daly MJ. Genome-wide association analysis identifies loci for type 2 diabetes and triglyceride levels. Science 2007;316: 1331-1336.

[104] Zeggini E, Weedon MN, Lindgren CM, Frayling TM, Elliott KS, Lango H, Timpson NJ, Perry JR, Rayner NW, Freathy RM. Replication of genome-wide association signals in UK samples reveals risk loci for type 2 diabetes. Science 2007;316: 1336-1341.

[105] Sladek R, Rocheleau G, Rung J, Dina C, Shen L, Serre D, Boutin P, Vincent D, Belisle A, Hadjadj S. A genome-wide association study identifies novel risk loci for type 2 diabetes. Nature 2007;445: 881.

[106] Scott LJ, Mohlke KL, Bonnycastle LL, Willer CJ, Li Y, Duren WL, Erdos MR, Stringham HM, Chines PS, Jackson AU. A genome-wide association study of type 2 diabetes in Finns detects multiple susceptibility variants. Science 2007;316: 1341-1345.

[107] Frayling TM. Genome-wide association studies provide new insights into type 2 diabetes aetiology. Nature Reviews Genetics 2007;8: 657.

[108] Durner M, Keddache MA, Tomasini L, Shinnar S, Resor SR, Cohen J, Harden C, Moshe $\mathrm{SL}$, Rosenbaum D, Kang H. Genome scan of idiopathic generalized epilepsy: evidence for major susceptibility gene and modifying genes influencing the seizure type. Annals of neurology 2001;49: 328-335.

[109] Ko Y, Cho M, Lee J-S, Kim J. Identification of disease comorbidity through hidden molecular mechanisms. Scientific reports 2016;6: 39433. 
Figure Legends

Figure 1: Study selection flowchart for the epilepsy and T2DM scoping review. 
Table 1: Search strategy

\begin{tabular}{|c|c|c|}
\hline Database & Search Strategy & Number of Articles \\
\hline $\begin{array}{l}\text { PubMed - first } \\
\text { attempt }\end{array}$ & $\begin{array}{l}\text { ("epilepsy"[TIAB] OR “antiepileptic"[TIAB]) AND } \\
\text { ("comorbidity"[TIAB] OR “comorbidities"[TIAB] OR } \\
\text { "type } 2 \text { diabetes"[TIAB] OR "obesity"[TIAB] OR } \\
\text { "obese"[TIAB] OR “exercise"[TIAB]) }\end{array}$ & 3,361 \\
\hline $\begin{array}{l}\text { PubMed - second } \\
\text { attempt }\end{array}$ & $\begin{array}{l}\text { "epilepsy comorbidity"[TIAB] OR "epilepsy } \\
\text { comorbidities"[TIAB] OR ("epilepsy"[TIAB] AND } \\
\text { "exercise"[TIAB]) OR ("epilepsy"[TIAB] AND "type } 2 \\
\text { diabetes"[TIAB]) OR ("epilepsy"[TIAB] AND } \\
\text { "obesity"[TIAB]) OR ("antiepileptic"[TIAB] AND } \\
\text { "exercise"[TIAB]) OR ("antiepileptic"[TIAB] AND } \\
\text { "obesity"[TIAB]) OR ("antiepileptic"[TIAB] AND "type } 2 \\
\text { diabetes"[TIAB]) }\end{array}$ & 1,008 \\
\hline Medline & $\begin{array}{l}\text { ("epilepsy comorbidity" or "epilepsy comorbidities" or } \\
\text { ("epilepsy" and "exercise") or ("epilepsy" and "type } 2 \\
\text { diabetes") or ("epilepsy" and "obesity") or } \\
\text { ("antiepileptic" and "exercise") or ("antiepileptic" and } \\
\text { "obesity") or ("antiepileptic" and "type } 2 \\
\text { diabetes")).ti,ab,cl,oa,kw,kf. }\end{array}$ & 851 \\
\hline
\end{tabular}




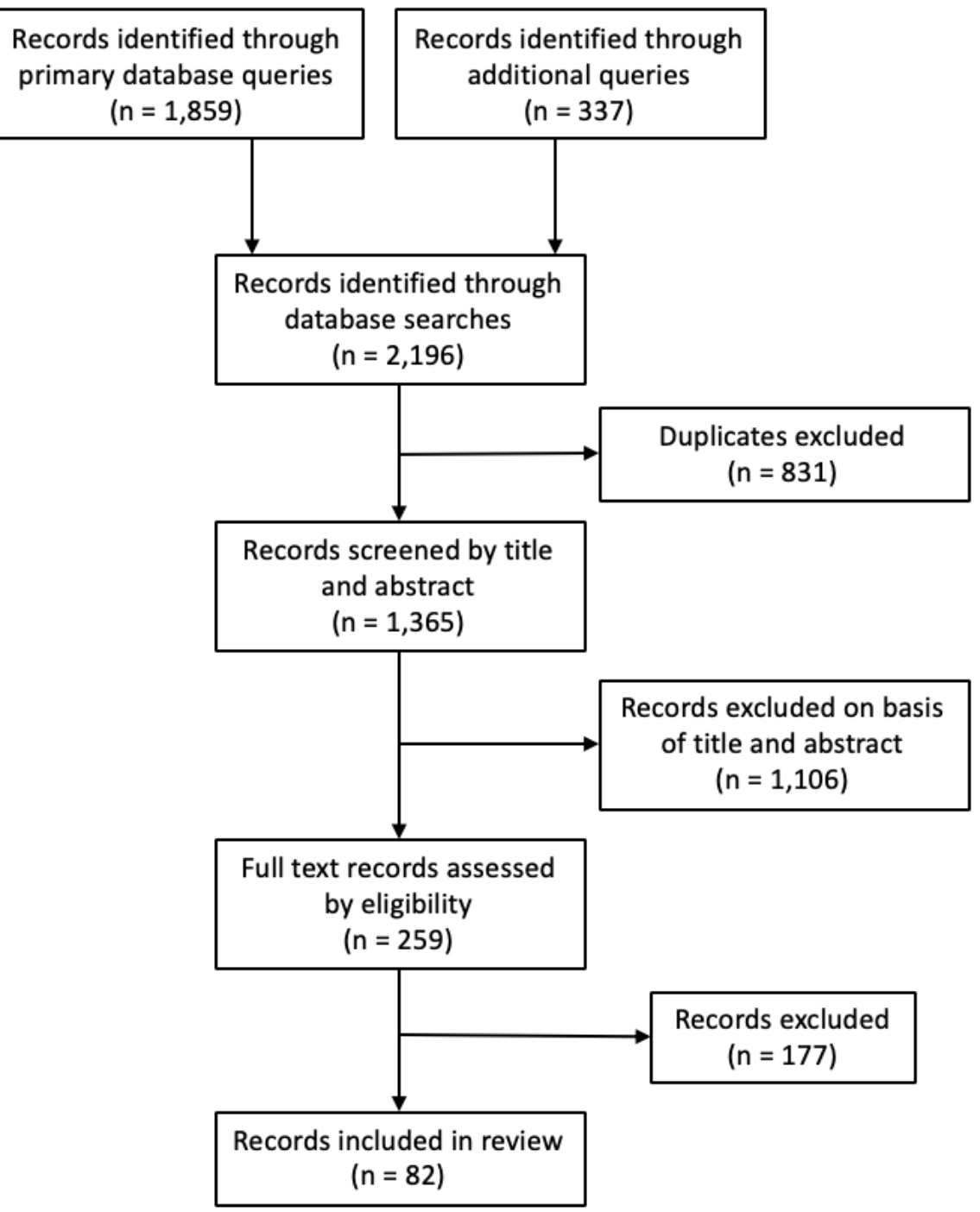

